\title{
Participación de los padres de familia en la escuela. La violencia como mediadora de la relación entre los padres y la escuela
}

Parent involvement in school. Violence as a mediator of the relationship between parents and school

Lina Paola Martínez Nieto Psicóloga, terapeuta familiar y orientadora escolar Colegio San Bernardino IED Secretaría de Educación Distrital de Bogotá. linapaolamn@gmail.com

Artículo de reflexión

Fecha de recepción: Junio 13 de 2015• Fecha de aprobación: Julio 26 de 2015

\section{ResUmeN}

El artículo reflexiona sobre cómo los procesos de participación de los padres de familia en la escuela, expresados en las formas de relación que se establecen entre ellos, muestran isomorfismo con las dinámicas de violencia que han caracterizado histórica y culturalmente a la sociedad de nuestro contexto colombiano. El análisis se fundamenta en la legitimación de dicha violencia, como medio de interacción social, a través de dispositivos de dominación en los ámbitos del cuerpo, el lenguaje y el espacio (Castillejo, 2009). Las ideas que se exponen en el artículo son fruto de algunos hallazgos iniciales de la investigación Representaciones sobre la participación de los padres de familia en la escuela, que la autora está desarrollando en el Colegio San Bernardino IED en Bogotá (Colombia), pero este no constituye un reporte de la investigación ni expone sus resultados, es un análisis de algunos aspectos identificados en el trabajo de campo que evidencian la presencia de dinámicas de violencia en el contexto de la escuela.

Palabras clave: violencia, participación, padres de familia, escuela. 


\begin{abstract}
The article reflects about how the parents' process of participation in the school, expressed in the ways of relationship that are established between them, shows isomorphism with the dynamics of violence which have characterized historically and culturally the society of our Colombian context. The analysis is founded on the legitimization of such violence, as a means of social interaction, throw the devices domination in the areas of the body, the language, and the space (Castillejo, 2009). The ideas that are exposed in the article are from the initial findings of the investigation "Representations about the parents' participation in the school", that the author is developing in the school "Colegio San Bernardino IED" in Bogotá, but this doesn't constitutes a report of the investigation neither expose it's results, is an analysis of some aspects identified on the field work that show the presence of violence dynamics on the school context.
\end{abstract}

Keywords: Participation, parents, school, violence. 


\section{INTRODUCCIÓN}

El artículo inicia su exposición contextualizando la investigación Representaciones sobre la participación de los padres de familia en la escuela, con el fin de enfocar al lector en las motivaciones de la misma, especialmente en la importancia de conocer los aspectos del funcionamiento escolar que interfieren en el establecimiento de relaciones cercanas y de colaboración entre los padres y la escuela, y en la pregunta por el papel que la violencia podría ejercer en dicho proceso. En el siguiente apartado se rescatan algunos elementos teóricos respecto a cómo se concibe la participación de los padres en la escuela, el impacto que esta puede generar en los procesos escolares, las condiciones que debe tener el sistema educativo para favorecerla y algunos obstáculos que varios autores han identificado. Acto seguido, se retoma el tema de la violencia y la dificultad de su conceptualización, se esbozan algunas definiciones y se delimita el análisis en la violencia simbólica. El siguiente apartado es el eje central del artículo, pues allí se tejen los hallazgos derivados de la investigación y el concepto de la violencia en los ámbitos del cuerpo, el lenguaje y el espacio. Se finaliza el texto con el apartado de conclusiones dando respuesta positiva a la hipótesis planteada en la reflexión.

\section{¿EN QUÉ CONTEXTO NACEN LA INVESTIGACIÓN Y EL ARTÍCULO?}

En el Colegio San Bernardino IED, que se encuentra ubicado en Bogotá (Colombia), se ha presentado una inquietud reiterativa, no resuelta, sobre las causas de la baja colaboración y participación de los padres en los procesos escolares, especialmente en el apoyo de las actividades de los estudiantes; pues además se observa una tendencia a la disminución progresiva de dicha participación o vinculación a medida que los niños y jóvenes avanzan en grado escolar.

Así mismo, se consideró pertinente investigar sobre el tema, pues aquellos estudiantes cuyos padres buscan comunicarse frecuentemente con los docentes, solicitan reuniones, establecen una relación cercana con el colegio, realizan acompańamiento y supervisión de tareas, colaboran en eventos escolares y participan en consejos o comités, muestran mejores resultados académicos (calificaciones, habilidad de 
análisis y comprensión), son más estables y cuentan con mejores habilidades para la socialización y la convivencia escolar, en comparación con aquellos estudiantes cuyos padres no generan estas acciones.

De hecho, la participación y el acercamiento de los padres con la escuela (en todas las acciones descritas en el párrafo anterior) muestran influencia positiva en los resultados de los estudiantes, idea que es respaldada desde ámbitos teóricos e investigativos (Cornejo y Redondo, 2007; Dabas, 2007; Epstein, 2005; Forest y García, 2006; Gerstenfeld, Franssen, Salinas, Cerda y Gómez, 1995). La mayoría de autores coindicen en plantear que el trabajo conjunto entre comunidad, hogar (padres, familiares) y colegio al apoyar los procesos escolares y de formación de los estudiantes, promueve que ellos se desarrollen armónicamente y que sus resultados de aprendizaje sean significativos.

De igual forma, el interés en el tema se fortaleció al observar que en la cotidianidad del colegio se manejan diversas "explicaciones" (informales) sobre la baja participación escolar de los padres. Es común escuchar comentarios como: "para los papás el colegio es una guardería”, "no les interesa apoyar a sus hijos con las tareas o asistir a las reuniones", "esperan que les terminemos de criar a sus hijos”. Fue así, que al no contar con evidencia documentada que sustentara estas ideas, se terminó de consolidar la propuesta de indagar sobre los elementos que favorecen u obstaculizan que los padres se vinculen activamente al colegio.

Entonces, se construyó la pregunta de la investigación: ¿cuáles son las representaciones, de padres y docentes, respecto a la participación de los primeros en la escuela?, pretendiendo esclarecer y describir la perspectiva de estos dos importantes actores de la comunidad educativa sobre por qué se presenta esa relación distante de los padres con el colegio. Grosso modo la investigación pretende identificar las representaciones, de padres y docentes, sobre: 1) vías formales de participación para los padres (representación de curso, comité de convivencia, consejo directivo, otros comités, etc.), 2) vías informales de participación para los padres (reuniones con docentes, charlas cotidianas, talleres, celebraciones, apoyo escolar de los estudiantes, etc.), 3) procesos y medios de comunicación en tre colegio y familias, y 4) elementos de orden sociodemográfico que puedan incidir en la 
disposición de los padres para vincularse al colegio. Con dichas representaciones se pretende identificar los elementos que fomentan u obstaculizan la disposición de los padres para vincularse al colegio (no se amplía información respecto al diseño y metodología de la investigación puesto que excedería los objetivos del presente artículo de reflexión).

Fue justo en el desarrollo del trabajo de campo de esta investigación, donde se identificaron ideas tanto de los padres como de los docentes sobre la presencia de condiciones o características del funcionamiento de la institución que dificultan el establecimiento de una relación estrecha entre el colegio y los padres de familia, las cuales tenían un elemento común: mostraban la hegemonía del manejo del poder desde adentro de la institución, especialmente de docentes, directivos y personal administrativo, dejando pocas posibilidades de involucramiento a los papás, a través de dinámicas de relación donde se descalifica la potencialidad o capacidad del padre para asumir un rol propositivo en el colegio. Es en esas dinámicas donde justamente se encontraron elementos de violencia simbólica, como se verá más adelante.

En el contexto de esta investigación surgió la motivación de construir el presente artículo de reflexión, enfocando el análisis en elementos de la experiencia directa de la investigadora tanto en el contexto educativo como en el contexto social de nuestro país, para lo cual se platearon las siguientes preguntas orientadoras: ¿de qué forma la presencia histórica de la violencia en Colombia se refleja en, y es mediadora de, las relaciones entre los padres y la escuela? ¿La violencia en dichas relaciones promueve la baja participación de los padres en el colegio? Esta no fue una inquietud amarillista ni perseguía una aprobación sentimentalista $y$, mucho menos, por considerarse su "pertinencia social" en términos de agenda pública; tan solo buscaba hacer un análisis juicioso sobre algunos elementos de la escuela que son cotidianos pero que pasan desapercibidos fácilmente y muchas veces se consideran "normales" o "legítimos" (por ejemplo, las ideas de los docentes sobre por qué los padres no se vinculan al colegio, el manejo del conducto regular, etc.), sin advertir que podrían configurar dinámicas de relación violentas. Por eso a través de los apartados que se presentan adelante, se espera evidenciar que la violencia sí se ha logrado adentrar en esta importante relación entre los padres y la escuela. 


\section{¿QUÉ HAN PLANTEADO LOS TEÓRICOS SOBRE LA PARTICIPACIÓN DE LOS PADRES EN LA ESCUELA?}

Los estudios sobre las escuelas eficaces han identificado que el éxito académico de los estudiantes está estrechamente relacionado con el trabajo mancomunado entre la familia y la escuela. Purkey y Smith (1983, citados por Parra, 2004) y Scheerens y Bosker (1997, citados por Parra, 2004) refieren que el apoyo y participación de la comunidad y los padres en la labor educativa son indicadores de calidad educativa y de resultados favorables de los estudiantes.

Según Kñallinsky (1999) la relación entre estas dos instituciones ha pasado por varias etapas. En la primera época, el Estado era el responsable de impartir y transmitir los conocimientos, apareció la escuela pública con la que los padres no tenían una relación directa. En la segunda época, se distribuyeron las cargas, la familia debía transmitir los valores (especialmente las madres) y la escuela instruía en contenidos específicos. En los años 60, hubo una etapa de la colaboración, la familia asumía la realización de actividades de apoyo escolar pero el poder continuaba depositado en el colegio, pues era allí donde se definían las formas concretas en las que la familia podía involucrarse. Los años 70 fueron una época de transición donde la relación se caracterizaba porque la escuela informaba a la familia. Y finalmente, desde la pedagogía, se consolidó “teóricamente" una época de la "participación” donde se planteó la necesidad imperante de que la escuela y la familia construyeran lazos cordiales, de apoyo mutuo y de cooperación real (Montessori, 1986; Freinet, 1976; Decroly y Monchamp, 1983; Dewey, 1967). Este último modelo exige horizontalidad en la relación con el poder equilibrado y distribuido, que se propongan formas novedosas de interacción y se generen canales alternativos de vinculación de la familia en los procesos escolares. Así, aparece la noción de coresponsabilidad sobre el proceso escolar de los estudiantes (Alcalay, Milicic y Torretti, 2005; Fernandez, 1992; Martínez, 2012; Parra, 2004; Pérez, 2001; Rodríguez, 2005; Tenti, 2004), haciendo fundamental una comunicación fluida y de doble vía, el respeto, la confianza y la actitud positiva ante el acercamiento activo de las familias en la escuela.

$\mathrm{Al}$ respecto, muchos autores coinciden en que la idea de la participación en el contexto escolar ha ganado gran aceptación. Fernández (1992) afirma que para los diferentes 
sectores de la comunidad escolar la participación es aceptada y casi indiscutida pero que está muy indefinida, por lo cual es susceptible de múltiples interpretaciones y connotaciones dependiendo del actor que la genere (docentes, directivos, padres, política pública, estudiantes), lo que la hace susceptible de conflictos, resistencias o predisposición. Por ello, señala que la participación requiere de un sistema educativo más horizontal, abierto a la comunidad y dispuesto a permitir el intercambio de información, saberes y estrategias, con confianza en todos los actores independiente de su nivel de formación y que sea un sistema que vaya más allá de la instrucción en contenidos, formando a los estudiantes para la vida en sociedad como ciudadanos y sujetos de derecho.

Por otra parte, Tenti (2004) afirma que los sistemas educativos se han consolidado desde una lógica homogenizadora, con relaciones jerárquicas y tendencia al manejo de límites muy cerrados, con carácter "sagrado", obstaculizando su interacción abierta y fluida con el entorno. Considera que dadas las condiciones de precariedad económica y social, de exclusión, de dominación-desequilibrio social y de debilidad estatal, la escuela requiere del apoyo de nuevos actores para lograr sus objetivos (los padres de familia).

Sin embargo, a pesar de la "aceptación" escolar y social sobre la importancia de la participación escolar de los padres, los autores plantean que existe gran dificultad para determinar de qué forma, en qué grado y bajo qué condiciones debe consolidarse dicho proceso. En este sentido Alcalay, Milicic y Torretti (2005) plantean que aún no se cuenta con suficiente claridad (sociocultural-investigativa) sobre las directrices de la participación de los padres en la escuela, pero plantean que debe fundamentarse en la construcción de un "vínculo" de la familia hacia la escuela, lo que implica generar un clima escolar de colaboración, cercanía y disponibilidad entre ambos, que favorezca también el "apego del estudiante con su colegio”. Sin embargo, las autoras plantean que:

Un aspecto que obstaculiza el compromiso familiar con la escuela y que hace que los padres eviten involucrarse, es la percepción por parte de estos de un clima escolar - la atmósfera social y educacional de la escuela- que no hace sentirse a las familias bienvenidas, respetadas, confiadas, escuchadas y 
necesitadas (...). En la medida que las escuelas crean un clima escolar positivo al acercarse a las familias y proporcionarles estructuras para que puedan involucrarse, el resultado es una alianza escuela-familia, efectiva (p. 151).

Siguiendo a Alcalay et al. (2005), las relaciones familia-escuela se caracterizan por el desconocimiento, la incomunicación y el desencuentro, pues ambas manejan percepciones erradas y realizan ejercicios de atribución mutua de la responsabilidad de las dificultades que exhiben los estudiantes. Lo anterior, se agudiza por la tendencia de la escuela a buscar la presencia de los padres, especialmente, cuando deben informarles sobre los problemas de los hijos, lo que genera una reacción crítica y defensiva de parte de ellos, caracterizada por "sentirse acusados, desvalorizados e inculpados", por lo que reaccionan externalizando la "culpa" y señalando que la escuela es "inadecuada e incompetente" para educar a su hijo. Entonces se consolidan imágenes negativas mutuas que abren cada vez más la brecha entre la familia y la escuela.

Como ha podido notarse, la participación escolar de los padres es considerada muy importante, pero los autores han identificado elementos que la obstaculizan, particularmente en el vínculo establecido entre ambas instituciones. De hecho, como lo veremos en los siguientes apartados, en la investigación sobre las Representaciones, de padres y docentes, sobre la participación escolar de los padres, se observaron algunos de estos elementos, y en particular, aquellos que evidencian aspectos de violencia que podrían ocupar un lugar explicativo sobre el impase que teóricamente vive la participación escolar de los padres.

\section{Y AL HABLAR DE VIOLENCIA EN LA RELACIÓN, ¿A QUÉ SE HACE REFERENCIA?}

Al hablar de violencia es inevitable el ingreso a un espacio caracterizado por la heterogeneidad y la relatividad, pues esta ha sido objeto de abordajes e interpretaciones desde diversas disciplinas y en varios contextos de análisis. La violencia ha sido estudiada por politólogos, sociólogos, psicólogos, economistas, antropólogos, 
criminalistas, violentólogos, entre otros, y entendida desde diversas dimensiones como la biológica, la sociológica, la política, la simbólica, la cultural, etc.

Blair (2009) refiere que en un intento de construir un concepto sobre la violencia que le permitiera abordar diversas de sus manifestaciones en la violencia colombiana, realizó una búsqueda entre los años de 1989 y 2009 tanto en literatura europea como latinoamericana, encontrando un panorama bastante complejo:

\begin{abstract}
Creía, entonces, que la posibilidad de consultar autores -no directamente colombianos, o con la gama de posibilidades de una universidad en un país europeo que en la época de auge de su política colonizadora se había paseado por los "violentos" países del llamado Tercer Mundo- podía permitirme abarcar un panorama mayor y construir conceptos más y mejor desarrollados teóricamente. No lo logré. Pero, al parecer, ni el intento ni el fracaso fueron solo míos, a juzgar por esfuerzos similares de otros académicos que, trasegando por diferentes recorridos, se enfrentaban a la misma dificultad (p. 1).
\end{abstract}

Mientras algunos consideran que la violencia es un fenómeno asociado a la política y al manejo del poder a través del uso ilegítimo o ilegal de la fuerza (Pécaut, 2013; Weber, 1984); otros lo relacionan con los conflictos bélicos, los enfrentamientos armados y las guerras (Arendt, 2005); mientras que desde otras perspectivas esta es entendida como un instinto agresivo humano derivado de nuestra historia genética (Lorenz, 1971); contrario a quienes la consideran un comportamiento aprendido en forma de actitudes de hostilidad; y finalmente, no puede obviarse la visión de la violencia como un fenómeno social simbólico y cultural (Bourdieu, 1999; Galtung, 2003).

A pesar de esta pluralidad, y sin deslegitimar alguna de las posturas teóricas, no se pueden eludir algunos aspectos fundamentales en el análisis de la violencia: 1) es un fenómeno específicamente humano, 2) no puede hablarse de una única violencia lo que determina la existencia "violencias", 3) es un fenómeno multicausado y polimorfo, y 4) dado su relativismo histórico su estudio requiere de un abordaje contextualizado histórica, social, política y culturalmente (Blair, 2009). Por lo anterior, el artículo aterriza sus análisis en los hallazgos de la investigación, dando cuenta de la influencia de la violencia dentro del escenario específico de las relaciones 
entre los padres de familia y el Colegio San Bernardino IED, lo cual no disminuye su potencial para hacer evidente un fenómeno que podría ocurrir también en otras instituciones educativas, consolidándose como un recurso importante en el campo de reflexión sobre las dinámicas escolares.

Para el artículo se asumirá la concepción de la violencia como un fenómeno construido socialmente, en el cual se generan una serie de mediaciones y transacciones simbólicas, y donde, por ende, la cultura juega un papel preponderante, para ello se retoman dos conceptos: violencia cultural y violencia simbólica.

Para Galtung (2003), violencia son todas aquellas amenazas que impiden a los seres humanos suplir sus necesidades básicas (supervivencia, bienestar, identidadrepresentación, y libertad) incluso hasta el punto de perder la vida. Propone el triángulo de la violencia evidenciando las caras o facetas que esta puede adquirir. Por un lado se encuentra la violencia directa, entendida como todo acto o acontecimiento que atenta contra las personas o las colectividades, cuyos efectos son fácilmente visibles o identificables (materiales, físicos), pero que también muestran huellas en términos emocionales o relacionales. Por otro lado, describe la violencia estructural o indirecta, que consiste en procesos o estructuras que provocan que la realización de las personas esté por debajo del lugar en el que podrían estar (privación de derechos, jerarquías, modos de trabajo, represión, fragmentación social), finalmente habla de la violencia cultural, que son todos aquellos aspectos simbólicos constantes o permanentes de una cultura que pueden usarse para legitimar las otras dos clases de violencia (normas de conducta, tradiciones, principios, arte, religión, lenguaje), así como para reprimir el sentido de defensa de quienes la sufren y justificar la destrucción y violencia mutua entre personas.

Llevando estos conceptos a la observación de una institución como la escuela, se puede sospechar que como todo grupo humano podría presentar situaciones de conflicto (o de contradicción en términos de Galtung, 2003) y, por ende también, situaciones de violencia. De hecho, la escuela cuenta con instancias y recursos que cumplen con características similares a las descritas en las tipologías de violencia; por ejemplo, manuales de convivencia, principios institucionales, estructuras jerárquicas, poder centralizado, entre otras, las cuales podrían consolidar dinámicas violentas 
como lo veremos en el siguiente apartado, pero centrando el análisis en el concepto de violencia simbólica.

Este concepto ha sido ampliamente abordado por Bourdieu y Wacquant (1995) cuando analiza las motivaciones que llevan a un grupo humano o a una sociedad a aceptar implícitamente situaciones de dominación y de manejo autoritario del poder desde clases sociales hegemónicas. Para ello propone la noción de violencia simbólica, resaltando el papel del lenguaje, los códigos y los significados en el mantenimiento de este tipo de formas de dominación en la existencia humana-social, los cuales no escapan a los más variados acontecimientos culturales y sociales: familia, educación, clases sociales, política, etc.

Entonces, se interpretará el ámbito de la relación de los padres de familia y la escuela desde esta noción de violencia simbólica, sin perder de vista que esta tiene una relación estrecha e interactuante con los demás tipos de violencia descritos anteriormente, como se verá a continuación.

\section{¿Cómo INTERVIENe La VIOLENCIA EN LAS RELACIONES PADRES-ESCUELA?}

Es necesario partir del hecho de que la violencia ha sido legitimada históricamente en aquellas sociedades que, como la colombiana, han transitado por procesos de colonización. Para Castillejo (2009), lo anterior se ha fundamentado en el mantenimiento de un dispositivo de dominación en tres ámbitos: Espacio-CuerpoLenguaje, donde las concepciones dicotómicas sobre el "yo" y los “otros”, como "el bueno" y "los malos" han atravesado las relaciones sociales determinando dinámicas de poder y de clase que "justifican" la violencia "restaurativa" de unos actores sociales sobre otros.

Las instituciones -familia y escuela- están inmersas en contextos sociales, culturales, políticos y económicos específicos, por ende, no están exentas de procesos históricos y de simbolización que determinan los roles y funciones que asumen en la educación; y que además provienen de un devenir del tiempo caracterizado por una marcada 
influencia de la violencia en nuestro país, por lo tanto, el Colegio San Bernardino IED no tendría por qué ser ajeno a esta realidad.

$\mathrm{Al}$ respecto y retomando elementos de los conceptos de violencia cultural (Galtung, 2003) y de violencia simbólica de Bourdieu y Wacquant (1995), autores que han analizado la violencia y el terror en países colonizados como Colombia (Blair, 2004; Taussig, 2002) han evidenciado la forma en que sistemáticamente se han construido lenguajes y procesos de simbolización que polarizan diversos ámbitos de la vida social. Por ejemplo, Chaves (2011) retomando y conciliando los planteamientos que sobre la corporalidad tienen los autores Maurice Merleau-Ponty, Marcel Mauss y Pierre Bourdieu, señala que en la sociedad se han construido dos lugares sociales en los que se ubican a las personas:

Para el análisis que aquí se propone, se destaca en qué dirección las categorías "personaje", "persona” y "yo" le otorgan al individuo la propiedad de tener un "papel" dentro de, ya sea un espacio sagrado o un espacio social; así, por ejemplo, la categoría "esclavo" no podría considerarse como sinónimo de "persona", puesto que "no tiene cuerpo, ni antepasados, ni nombre, ni cognomen, ni bienes propios (Chaves, 2011, p. 166).

Con base en estas ideas, socialmente habría "unos" que sí lograrían el estatus de persona, merecedores de dignidad, respeto y reconocimiento de un lugar en la sociedad, mientras que los "otros" no, al carecer de las "condiciones" necesarias. Estos roles han sido construidos con base en la tenencia histórica de una posición de poder o de hegemonía. En Colombia esta situación proviene de siglos atrás cuando fuimos denominados "Salvajes" e "Incivilizados" por los conquistadores-colonizadores al no poseer su mismo "desarrollo", su misma estructura social, su mismo idioma y su misma forma de operar socialmente, de manera que éramos "el Nuevo Mundo donde los "irracionales", indios y africanos se inclinan ante la razón de un reducido número de cristianos blancos" (Taussig, 2002, p. 26). Blancos que pretendieron y asumieron el estatus de civilización y sabiduría de la que supuestamente carecíamos, y que a través de diversos dispositivos de dominación y violencia (de todo tipo) nos "amaestraron" instruyéndonos en lo que "debíamos llegar a ser". 
Esta dinámica se introdujo en lo más profundo de nuestro funcionamiento social y aun hoy, prevalecen las categorizaciones, clasificaciones y segmentaciones de los lugares o clases sociales. Entonces, como lo plantearon implícitamente algunos autores en apartados previos, al revisar el contexto de la escuela se evidencian claras diferencias en las nociones "docente" y "padre de familia", pues están asignados a posiciones y ámbitos sociales distintos, casi excluyentes. Siguiendo la metáfora, así como la categoría de "esclavo" no era sinónimo de "persona” pues no tenía nombre, ni bienes propios, los padres no podrían considerarse legítimos agentes educativos porque no poseen "nombre profesional" ni "bienes de formación académica". De hecho en la investigación se encontraron planteamientos de padres y docentes respecto a que la baja formación o nivel educativo no favorece su participación en el colegio y afecta negativamente su iniciativa; de hecho, los docentes consideran que los padres no cuentan con suficientes elementos para opinar sobre los aspectos netamente pedagógicos, lo cual constituye lugares de poder y participación diferentes en el proceso escolar de los estudiantes.

Por otro lado, aunque tanto nacional como distritalmente se han generado políticas públicas que resaltan la pertinencia de la participación de los padres en la escuela (Constitución Política de Colombia 1991, artículos 67 y 103; Ley 134 de 1994; Código de la Infancia y la Adolescencia, Ley 1098 de noviembre 8 de 2006, artículo 39, numerales 8 y 9; Ley general de educación 115 de 1994, artículos 6, 7, 142, 143 y 164; Decreto 1286 del 27 de abril de 2005; Ley 1620 del año 2013, artículo 12), en la práctica esto no se evidencia en su implementación, pues al hacer revisión de documentos y literatura se nota escasa evidencia de iniciativas sistemáticas y suficientes de la escuela para incentivar la participación de los padres (MEN, 2001). Por el contrario, pareciera que la escuela se ha blindado simbólicamente a través de prejuicios y conclusiones ligeras y culpabilizadoras (ni intencionadas ni conscientes en muchas ocasiones) como se evidencia en el colegio con las "explicaciones informales" sobre la pobre vinculación de los padres a los procesos escolares; así, los padres se configuran como los "malos" y los docentes como las "víctimas" de las irresponsabilidades parentales, lo que constituye una actitud violenta en esa relación, generada probablemente por la normalización o legitimación de dichas ideas y por el desconocimiento de esa alteridad que son los padres y sus experiencias de vida. 
Con base en lo anterior, podría considerarse que se ha consolidado una dinámica de violencia simbólica en la relación padres-escuela, pues con el fundamento de interpretaciones legitimadas gracias a su constante repetición, se desdibuja la potencialidad de escuchar a los padres, de conocerlos, de saber qué tipo de educación desean para sus hijos y, especialmente, de saber por qué no se involucran en el colegio. Esto probablemente por la imposición histórica de unos "dispositivos hermenéuticos", que rezan que a las "minorías" no se les escucha porque no tienen ni siquiera el estatus de "persona", y que como los padres no tienen altos niveles de formación entonces "no saben". Estas dinámicas se han ido incorporando de tal manera que ni los padres muestran siquiera la intención de involucrarse en los asuntos de la escuela, en tanto se sienten incapaces e ignorantes, y tampoco muestran la iniciativa de defenderse de tales simbólicos atropellos.

Al respecto, Van Dijk (2004) con el Análisis critico del discurso, plantea que nuestras sociedades han estado sometidas al influjo y abuso del poder por parte de grupos e instituciones dominantes, que se valen del lenguaje y del discurso para ejercer control sobre el funcionamiento social, manteniendo la desigualdad e inequidad social. Aunque ese poder puede ser físico-corporal como en los enfrentamientos armados o en la violencia de género (física, psicológica, social), hace más referencia es a un poder del discurso:

El poder discursivo es más bien mental. Es un medio para controlar las mentes de otras personas y así, una vez que controlemos las mentes de otros, también controlamos indirectamente sus acciones futuras. En este caso no necesitamos forzar a las personas para que hagan algo, sino que ellas hacen lo que queremos en su libre albedrío o bien porque no tienen alternativas. De esta forma es que podríamos manipular, informar mal, educar mal, etc., a otras personas de acuerdo con nuestro interés y en contra de sus más altos intereses (...). El poder está basado en recursos sociales escasos como dinero, tierras, casas, un buen salario y otros recursos materiales; o en conocimiento, fama, cultura y recursos simbólicos similares. Uno de estos recursos es el acceso preferencial al discurso público (Van Dijk, 2004, p. 9). 
En el funcionamiento escolar se evidencia claramente que el discurso de los intelectuales de la educación, con su respaldo científico e investigativo, es hegemónico sobre lo que una persona "común y corriente" pudiese opinar en cuanto a la escuela, su proyección o su funcionamiento pedagógico. Según los hallazgos de la investigación, los padres, en su mayoría, tienen acceso limitado a dicho poder discursivo, generalmente sus palabras son minimizadas al "no poseer grandes recursos materiales ni procesos de formación académica”, lo cual por ende es un funcionamiento violento que ha terminado siendo tan incorporado que se asume como natural o normal.

Entonces, ¿qué hay en el discurso público?, ¿quiénes sí acceden a él? Por supuesto que los que sí pueden son los "personajes de la vida pública" como los políticos, los intelectuales, los dueños de los medios de información masiva, los periodistas y los mismos docentes. Estos personajes lideran lo que podrían denominarse las "élites simbólicas". Por lo tanto, ni los trabajadores informales, ni los pobres, ni las amas de casa, ni los niños, entre otras "minorías" tienen el acceso a este espacio de control. De hecho, en el colegio investigado la mayoría de padres consideran que sus ideas y propuestas sobre los procesos escolares no son escuchadas y especulan que tal vez eso tiene que ver con opiniones de los profesores y directivos sobre sus "bajas capacidades".

Otro elemento que evidencia dicha violencia incorporada en las relaciones escuelafamilia, es el manejo del "cuerpo" o la "corporalidad", nótese cómo la misma estructura física del Colegio San Bernardino IED impide el acceso frecuente de los padres a las instalaciones, se les cierra la puerta cuando no tienen una citación formal proveniente de algún miembro de la comunidad educativa que permanezca dentro de estos límites físicos, es decir, los docentes y directivos. En esta dinámica, que constituye tanto violencia simbólica como una acción violenta directa, es evidente que el poder está centrado en pocas manos, que lo que ocurre en los procesos educativos es gestión de quienes están dentro del colegio y que implícitamente se les dice a los padres que "solo podrán involucrarse cuando desde adentro se les invite". 
$\mathrm{Al}$ respecto, es interesante retomar los planteamientos que Chaves (2011) hace sobre la visión de la corporalidad desde Bourdieu:

Debido a la corporeidad y a la incorporación, los habitus tienen la facultad de ser estructuras generadoras de prácticas. Sin embargo, la incorporación obedece a un proceso de "instrucción" que parte de una "necesidad social vuelta naturaleza”, es decir, de cómo los habitus son tomados como parte genética del individuo, no tanto porque se inscriban en sus códigos biológicos, sino por el temprano aprestamiento a los mismos. En segundo lugar, se incorpora lo que es socialmente aceptado, puesto que el cuerpo y su disposición tienen interés social en la medida en que obedecen al orden que puede ser evocado a través de las prácticas en un momento dado; finalmente, hay un sentido incorporado cuando este hace parte de la práctica cotidiana (p. 167).

Así, el manejo físico-corporal que se ha generado con los padres en el colegio, sumado a la dominación del discurso de la élite intelectual, ha podido estimular la incorporación tanto en los docentes como en los padres de una serie de prácticas que favorecen el distanciamiento de estos últimos con la escuela. Se considera obvio y es aceptada socialmente la idea de que los que tienen el conocimiento y las habilidades para enseñar son los docentes, que son ellos quienes pueden dar la última palabra sobre las necesidades de los estudiantes y el tipo de herramientas pedagógicas que se deben utilizar para enseñarles. Por lo tanto, las opiniones y sugerencias de los padres pierden toda validez y confianza en su utilidad, lo cual disminuye la tendencia a su involucramiento en los procesos escolares de sus hijos.

Es inquietante sobre este punto el hecho de que los docentes, teniendo amplios procesos de formación y de investigación, asuman estas actitudes de dominación, lo que cuestiona sobre dos aspectos: por un lado, hasta dónde también están inmersos en los discursos del poder que obstaculizan el análisis crítico y la capacidad de reconocimiento de las alteridades; y por otro lado, cómo la formación universitaria puede reproducir el sentimiento de pertenencia a la élite discursiva dominante.

Otro elemento del dispositivo de dominación que se ha venido tratando, es la noción de "espacio", lo que nos lleva directamente a pensar en el "territorio" (Castillejo, 
2009). Cuando un colegio se ubica en una zona periférica (semirural) como el barrio San Bernardino cuyo proceso de urbanización está apenas madurando pues hasta hace pocos ańos era una vereda, es factible que se les considere distintos, incultos, campesinos, pobres y poco preparados para participar activamente en la escuela, contrario a agentes educativos formados académicamente. De hecho en la investigación se encontraron referencias negativas de los profesores respecto a los padres y su "falta de cultura", a la "necesidad formarlos", que en las familias "no le dan importancia a la educación porque no es algo que venga de la formación de sus familias de origen", que así como los padres piensan que salieron adelante sin educación "el proyecto de vida de los hijos no es estudiar sino trabajar", las cuales denotan unas ideas sesgadas de la población pues, por el contrario, en las entrevistas con los padres se encontraron referencias numerosas y significativas sobre la importancia de la educación, el interés de que los niños vayan al colegio y de que reciban una buena formación.

Esta dinámica es similar a la descrita por Castillejo (2009) respecto a la violencia en Sudáfrica cuando explica cómo se construyen "enemigos" a través de la asociación de algunas minorías con nociones como la "amenaza” y el "peligro", para justificar la violencia hacia ellas con el aparente objetivo de restablecer el orden social: "...se articulan muy claramente nociones tales como "enemigo", "terrorista", "violencia", "peligro" y "lugar". En este contexto convergen discursos en torno al otro negro, la turba irracional, la "zona del desorden" y el conflicto" (p. 108). Llevando estos planteamientos a las dinámicas escolares en la investigación fue posible identificar que en ocasiones los docentes sienten amenazada su labor cuando los padres intentan "inmiscuirse" en sus estrategias pedagógicas, son "enemigos al acecho". Esto es evidente en la gran molestia que manifiestan cuando un padre o madre de familia hace alguna sugerencia sobre el manejo que considera que debe tener el docente en el aula, los docentes reaccionan por lo general a la defensiva y profiriendo comentarios desagradables respecto a los progenitores.

Por otro lado, en el territorio aledaño al colegio, donde se ubican las familias de los estudiantes, se presentan problemas de delincuencia común, expendio y consumo de sustancias psicoactivas, robos frecuentes y posible presencia de grupos armados (de civil). Sumado al hecho de que la zona es receptora de muchas personas 
desplazadas por la violencia o que migraron hacia las urbes en la búsqueda de mejores oportunidades sociales y laborales. Esta situación fomenta las asociaciones que propone Castillejo (2009) entre las categorías: sur, pobres, peligro, delincuentes, expendio, desplazados, etc., y los grupos minoritarios, lo que fortalece el discurso del poder y el empoderamiento de las élites intelectuales en los colegios, donde el Colegio San Bernardino no es la excepción, y entonces, se consolidan dinámicas de relación verticales, impositivas desde el discurso docente y donde se hace una negación implícita del padre de familia como un "otro válido" de quien se puede recibir realimentación.

Sumado a los autores del apartado anterior, Gil (1997) afirma que en la escuela se da un juego de fuerzas, de relaciones de poder entre los actores de la escuela y los roles que cada quien asume. Refiere que los profesores tienen una mayor participación, casi hegemónica, incluso sobre los estudiantes, al contar con la posibilidad de reunirse, de pertenecer a estamentos como consejos donde se establecen propuestas y se toman decisiones sobre el funcionamiento de la institución escolar. Confronta la auténtica intención y proactividad de los docentes para el establecimiento de estrategias concretas para la promoción de la participación de los padres, de hecho considera que utilizan, más bien, ejercicios de control justificados con atribuciones externas disfrazadas de explicación, tales como que los padres "no están preparados", "no ven la importancia de los consejos o comités escolares", "no tienen tiempo", "están cansados", "el colegio está lejos de su casa”, etc.

Respecto a lo anterior, en la investigación se observó que aunque los padres quisieran tener una mayor participación en el colegio, no reconocen claramente los mecanismos o vías a través de las cuales pueden establecer esa relación de cercanía, no saben para qué se eligen "padres representantes de curso", ni que tienen un lugar en el consejo directivo y el comité de convivencia, y aunque reconocen que en algunos colegios hay asociaciones de padres ni siquiera lo contemplan como una opción en el Colegio San Bernardino. De igual forma, los padres de familia niegan que el colegio fomente su participación, solamente identifican los talleres de escuela de padres (proyecto que tiene 4 años en ejecución) como espacios en los que pueden aprender y acercarse. Entonces, si los padres no reconocen las vías de participación ni hacen uso de ellas, es evidente que los horizontes institucionales 
son determinados por los actores que están dentro de los límites del colegio y que asumen activamente los roles y funciones determinados para ello, los docentes y directivos. Esta dinámica implícitamente constituye una relación vertical donde el poder está depositado en unas solas manos, el cual es un elemento de las dinámicas de violencia simbólica.

Por otro lado, contrario a lo que podrían suponer muchos docentes desde sus “explicaciones informales”, Gil (1997) encontró que los padres generalmente no participan porque están desinformados de la posesión de este derecho del que son poseedores y porque sienten fuerte temor de no contar con la formación, bagaje, destreza cognitiva, competencia discursiva y argumentativa para ejercer una "adecuada" participación, lo que permite evidenciar la incorporación del discurso dominante. Lo anterior se corroboró en las entrevistas con los padres, pues aunque expresaban tener el derecho a participar, no conocían las vías o mecanismos para hacerlo y expresaban gran inseguridad y temor de no poseer la capacitación y habilidad suficiente para involucrarse en los asuntos del colegio.

Otros autores también resaltan la necesidad de que los padres hagan presencia en la escuela pero describen que las dinámicas entre ambos son complejas. Por ejemplo, García-Bacete y Rustarazo (2005) refieren que generalmente los docentes manifiestan que los padres no son colaboradores ni asisten a las reuniones; y que los padres, por su parte, se quejan de que los profesores generalmente los llaman cuando "las cosas van mal" o les piden hacer cosas que "no saben hacer". Además, señalan que la falta de tiempo es un elemento que dificulta la colaboración escuela y familia, pero que la falla radica principalmente en la pobre comunicación, aparentemente, porque ni los padres ni los profesores cuentan con las habilidades para establecer procesos de empatía (ponerse en el lugar del otro).

Lo anterior es implícitamente una dinámica violenta, pues hay una descalificación mutua y ninguna de las partes muestra el interés de vincularse con la otra, se atribuyen recíprocamente la responsabilidad de los resultados de los estudiantes, guardan resistencias y desconfianza entre sí, dejando a la educación en un estado de bloqueo del cual no ha salido desde hace mucho tiempo. 


\section{AlgunAS CONCLUSIONES PARCIALES}

Los hallazgos iniciales de la investigación permitieron identificar evidencia significativa de que en la estructura y el funcionamiento del colegio, así como en el tipo de relaciones que se establecen entre este y los padres, hay variados elementos dentro de los ámbitos del "cuerpo", "el territorio" y "el lenguaje" (Castillejo, 2009) que constituyen dinámicas de violencia simbólica, los cuales a su vez, afectan la iniciativa de los padres para participar activamente en los procesos escolares.

Es así que se puede concluir, que las relaciones padres-escuela, están fuertemente manchadas por la legitimación histórica de la violencia, por la dominación de unas elites sobre la población en general, lo que ha generado fuertes rivalidades entre estos dos escenarios fundamentales del proceso educativo: la escuela y la familia, dejando vulnerables a niños, niñas y jóvenes que requieren de su trabajo mancomunado

Lo más doloroso del mantenimiento de estas dinámicas es que tanto los padres como los docentes sueñan y desean futuros promisorios para los estudiantes, pero ambos siendo víctimas de los dispositivos de dominación descritos terminan actuando su papel de "marionetas" de una historia ajena, escrita por otros para quienes sus propios intereses priman sobre los intereses sociales y para quienes el uso de la violencia es justificable como medio para la obtención de sus propios fines.

En definitiva, ni la escuela se logró escapar de la influencia de la violencia simbólica y cultural legitimada históricamente en el país. 


\section{Referencias}

Alcalay, L., Milicic, N. y Torretti, A. (2005). Alianza efectiva familia-escuela. Un programa audiovisual para padres. Psykhe, 14(2), 149-161.

Arendt, H. (2005). Sobre la violencia. Madrid: Alianza Editorial.

Blair, E. (2004). Mucha sangre y poco sentido: la masacre. Por un análisis antropológico de la violencia. Boletín de Antropología, 8(35), 165-184.

Blair, E. (2009). Aproximación teórica al concepto de violencia: avatares de una definición. Politica y Cultura, 32, (pp. 9-33).

Bourdieu, P. y Wacquant, L. (1995). Respuestas. Por una antropología reflexiva. México: Grijalbo.

Bourdieu, P. (1999). Meditaciones pascalianas. Barcelona: Editorial Anagrama.

Castillejo, A. (2009). Los archivos del dolor. Ensayos sobre la violencia y el recuerdo en la Sudáfrica contemporánea. Bogotá: Ediciones Uniandes.

Chaves, J. (2011). Entre la violencia sobre el cuerpo y la violencia incorporada. Hacia la Promoción de la Salud, 16(2), 162-172.

Cornejo, C. R. y Redondo, J. (2007). Variables y factores asociados al aprendizaje escolar. Una discusión desde la investigación actual. Estudios Pedagógicos, 33(2), 155-175.

Dabas, E. (2007). Compartiendo territorios: relaciones familia-escuela. En Dabas, E. (Comp.). Viviendo redes. Experiencias y estrategias para fortalecer la trama social. Buenos Aires: Ediciones CICCUS.

Decroly, O. y Monchamp, E. (1983). El juego educativo. Iniciación a la actividad intelectual y motriz. Madrid: Morata. 
Dewey, J. (1967). El niño y el programa escolar. Mi credo pedagógico. Buenos Aires: Losada.

Epstein, J. (2005). A case study of the partnership schools Comprehensive School Reform (CSR) model. The Elementary School Journal, 106(2), 151-170.

Fernández, M. (1992). Poder y participación en el sistema educativo. Sobre las contradicciones del sistema escolar en un contexto democrático. Barcelona, España: Paidós.

Forest, C. y García, J. F. (2006). Comunicación cooperativa entre familia y escuela. España: Nau Libres.

Freinet, C. (1976). Las invariantes pedagógicas. Guia práctica de la escuela moderna. Barcelona: LAIA.

Galtung, J. (2003). Violencia cultural. Gernika-Lumo: Gernika Gogoratuz.

García-Bacete, F. J., y Rustarazo, F. J. (2005). Cómo son y cómo podrían ser las relaciones entre escuelas y familias en opinión del profesorado. Comunicación presentada en las IV Jornadas de desarrollo humano y educación. Educar para el Cambio: Escenarios para el desarrollo humano. Universidad de Alcalá de Henares.

Gerstenfeld, P., Franssen, A., Salinas, A., Cerda, A. M., Edwards, V. y Gómez, M. V. (1995). Variables extrapedagógicas y equidad en la educación media: hogar, subjetividad y cultura escolar. Santiago de Chile: Naciones Unidas. Comisión Económica para América Latina y el Caribe.

Gil, F. (1997). La participación democrática en la escuela: De cómo los agentes educativos se las ingenian para gobernarse. Bogotá: Editorial Magisterio.

Kñallinsky, E. (1999). La participación educativa: familia-escuela. Gran Canarias: Servicio de Publicaciones de la Universidad de las Palmas.

Lorenz, K. (1971). Sobre la agresión: el pretendido mal. Buenos Aires: Siglo XXI Editores. 
Martínez, S. (2012). La relación familia-escuela. La representación de un espacio compartido. Tesis doctoral. Universidad de Barcelona.

Ministerio de Educación Nacional. (2001). Por una escuela más democrática. Al tablero: periódico de un pais que educa y que se educa, 3. Recuperado de http://www.mineducacion. gov.co/1621/article-87181.html

Montessori, M. (1986). La mente absorbente del niño. México: Biblioteca Editorial Diana.

Parra, J. M. (2004). La participación de los padres y la sociedad circundante en las instituciones educativas. Tendencias Pedagógicas, 9, (pp.165-187).

Pécaut, D. (2013). La experiencia de la violencia: los desafíos del relato y la memoria. Medellín: La Carreta Editores.

Pérez, T. (2001). Ciencia solidaria y democrática. Nuevos paradigmas y estrategias pedagógicas para su construcción. Bogotá: Ismac.

Rodríguez, E. G. (2005). Educación y educadores en el contexto de la globalización. Revista Iberoamericana de Educación, 35(6). Recuperado de http://www.rieoei.org/ deloslectores/910Rodriguez.PDF

Taussig, M. (2002). Chamanismo, colonialismo y el hombre salvaje. Un estudio sobre el terror y la curación. Bogotá: Editorial Norma.

Tenti, E. (2004). Notas sobre escuela y comunidad. Seminario internacional alianzas e innovaciones en proyectos educativos de desarrollo local. Buenos Aires: IIPE/Unesco.

Van Dijk, T. A. (2004). Discurso y dominación. Grandes conferencias en la Facultad de Ciencias Humanas, 4. Universidad Nacional de Colombia.

Weber, M. (1984). El politico y el cientifico. Madrid: Editorial Alianza. 